\title{
Rotational laser cooling of vibrationally and translationally cold molecular ions
}

\author{
Peter F. Staanum, Klaus Højbjerre, Peter S. Skyt, Anders K. Hansen and Michael Drewsen *
}

Stationary molecules in well-defined internal states are of broad interest for physics and chemistry. In physics, this includes metrology ${ }^{1-3}$, quantum computing ${ }^{4,5}$ and manybody quantum mechanics ${ }^{6,7}$, whereas in chemistry, stateprepared molecular targets are of interest for uni-molecular reactions with coherent light fields ${ }^{8,9}$, for quantum-stateselected bi-molecular reactions ${ }^{10-12}$ and for astrochemistry ${ }^{12}$. Here, we demonstrate rotational ground-state cooling of vibrationally and translationally cold $\mathbf{M g H}^{+}$ions, using a lasercooling scheme based on excitation of a single rovibrational transition ${ }^{13,14}$. A nearly 15 -fold increase in the rotational ground-state population of the $X^{1} \Sigma^{+}$electronic groundstate potential has been obtained. The resulting ground-state population of $36.7 \pm 1.2 \%$ is equivalent to that of a thermal distribution at about $20 \mathrm{~K}$. The obtained cooling results imply that cold molecular-ion experiments can now be carried out at cryogenic temperatures in room-temperature set-ups.

At present there is a strong interest within the scientific community to produce and experiment with cold molecules. As standard laser-cooling schemes developed for atomic species cannot be applied to molecules, in the recent past tremendous effort has been put into developing schemes to cool molecules by other means. Several schemes for producing translationally cold and strongly bound neutral molecules in a single quantum state have been demonstrated. These include buffer-gas cooling of magnetic dipolar molecules in magnetic traps ${ }^{15}$, Stark deceleration and trapping of molecules with permanent ${ }^{16,17}$ or induced $^{18}$ electric dipole moments, formation of molecules through Feshbach resonances followed by transfer to the vibrational ground state of electronic molecular potentials by stimulated Raman adiabatic passage schemes ${ }^{19}$ and photoassociative molecule formation directly in the rovibrational ground state ${ }^{20}$ or by transfer to the vibrational ground state through the application of shaped femtosecond laser pulses ${ }^{21}$.

With respect to molecular ions, the well-established technique of buffer-gas cooling is the only method that has so far led to the production of translationally as well as internally cold molecular ions ${ }^{22}$. Although buffer-gas cooling is simple and generally applicable, it limits both the effective internal and translational temperature to the kelvin range, and owing to frequent collisions with the buffer-gas atoms it prevents strong spatial localization as well as utilization of internal coherences. Sympathetic cooling of trapped molecular ions, through the Coulomb interaction with laser-cooled atomic ions, has, on the other hand, proven highly effective for reaching translational temperatures in the millikelvin range, where the ions become spatially localized in the form of so-called Coulomb crystals $^{23}$. The sympathetic cooling scheme does, however, not significantly influence the molecular ion's internal degrees of freedom, owing to the very distant Coulomb interactions within the crystalline structures. For polar molecular ions, this means that the rovibrational degrees of freedom on a timescale of 10-100 s will equilibrate to the temperature of the blackbody radiation (BBR) in the trapping region. Consequently, a significant population in a large number of rovibrational levels will result ${ }^{13,24,25}$. To solve this problem, several schemes have recently been proposed for internal state preparation of sympathetically cooled molecular ions $s^{13,14,26}$.

Following one of the rotational cooling schemes, here we report on rotational ground-state laser cooling of ensembles of trapped $\mathrm{MgH}^{+}$molecular ions that are already sympathetically cooled by $\mathrm{Mg}^{+}$ions to a translational temperature below $100 \mathrm{mK}$. Owing to the large vibrational constant of the $\mathrm{X}^{1} \Sigma^{+}$electronic ground-state potential of the $\mathrm{MgH}^{+}$ion, they are furthermore initially in the vibrational ground state ( $>99.9 \%$ probability).

In Fig. 1a, the scheme for rotational laser cooling towards the $|v=0, J=0\rangle_{\mathrm{X}}$ state is sketched. In brief, the scheme is based on a single laser excitation of the $|v=0, J=2\rangle_{\mathrm{X}}-|v=1, J=1\rangle_{\mathrm{X}}$ transition. This excitation effectively transfers population from the $|v=0, J=2\rangle_{\mathrm{X}}$ level to the $|v=0, J=0\rangle_{\mathrm{X}}$ ground state by spontaneous decay from the $|v=1, J=1\rangle_{\mathrm{X}}$ level, either directly to the ground state $|v=0, J=0\rangle_{\mathrm{X}}$ or back to the $|v=0, J=2\rangle_{\mathrm{X}}$ level from where it will be excited again. Although population pumped into the ground state will be transferred step-by-step towards higher lying levels owing to dipole-allowed rotational transitions $(\Delta J= \pm 1)$ induced by BBR, any time the population enters the $|v=0, J=2\rangle_{\mathrm{X}}$ level, it will rather efficiently be pumped back to the ground state. This 'Sisyphus'-mechanism combined with transfer of population from levels with $J>2$ towards the $|v=0, J=2\rangle_{\mathrm{X}}$ level through BBR-induced rotational state-changing transitions lead eventually to strong rotational cooling.

For determining the rotational state population of the various $|v=0, J\rangle_{\mathrm{X}}$ levels, a two-colour $1+1$ resonance-enhanced multiphoton dissociation (REMPD) technique is applied with the resonant intermediate level being one of the $|v=0, J \pm 1\rangle_{\mathrm{A}}$ levels of the $\mathrm{A}^{1} \Sigma^{+}$potential and dissociation through the $\mathrm{C}^{1} \Sigma^{+}$potential. As can be seen in Fig. 1b, dissociation through the $\mathrm{C}^{1} \Sigma^{+}$potential results in a neutral $\mathrm{Mg}$ atom and a proton, which is too light to be trapped. Consequently, a single REMPD event, defined as the simultaneous application of single pulses of the two colours, leads to a detectable loss in the number of trapped $\mathrm{MgH}^{+}$ions proportional to the population of the particular rotational state being addressed ${ }^{13,24}$

In the experiment, about $1,000 \mathrm{Mg}^{+}$ions are first trapped in a linear Paul trap and Doppler cooled using the $3 s^{2} \mathrm{~S}_{1 / 2}-3 p{ }^{2} \mathrm{P}_{3 / 2}$ transition to a translational temperature of around $10 \mathrm{mK}$. At these low temperatures the $\mathrm{Mg}^{+}$ions form a Coulomb crystal of which a two-dimensional projection image is presented in Fig. 2a (I). $\mathrm{Next}, \mathrm{MgH}^{+}$ions are formed through reactions between $\mathrm{Mg}^{+}$ions excited to the $3 p{ }^{2} \mathrm{P}_{3 / 2}$ state by the cooling laser beams and $\mathrm{H}_{2}$ molecules at room temperature, leaked into the vacuum chamber 
a

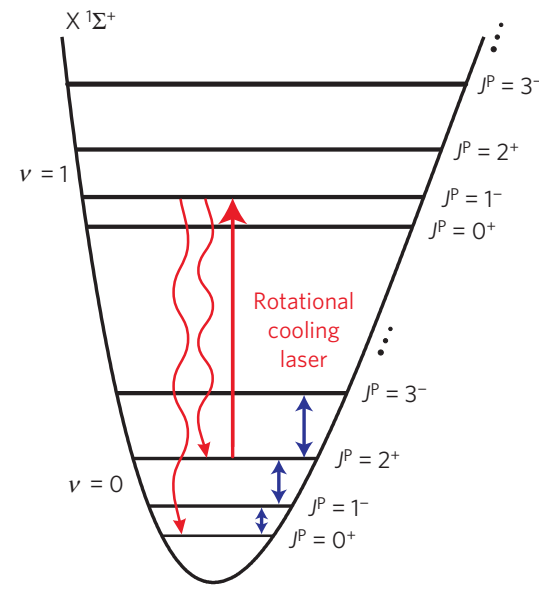

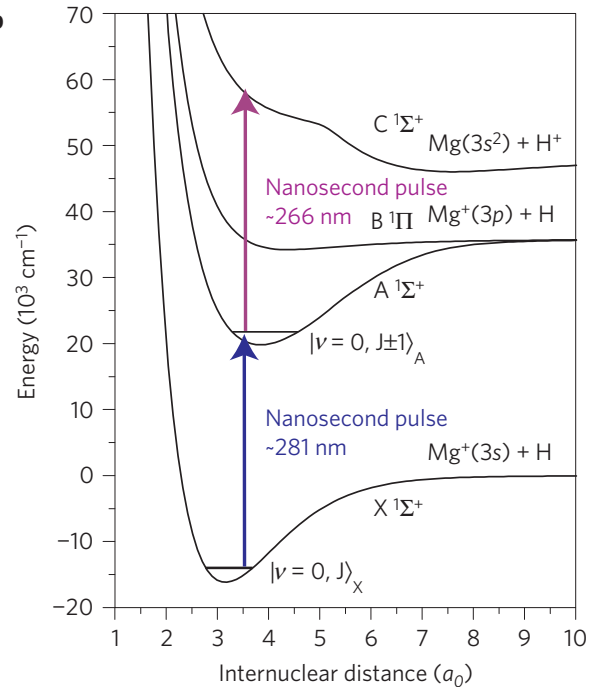

Figure 1 | Schemes for rotational cooling and for rotational state distribution determination. a, The main rovibrational states ( $v$ is the vibrational quantum number, $J$ is the rotational quantum number and $\mathrm{P}$ is the parity of a given state) and electric-dipole-allowed transitions (only $\Delta J= \pm 1$ owing to parity) involved in the rotational cooling scheme. The straight red arrow indicates laser excitation; wavy arrows represent possible spontaneous decay

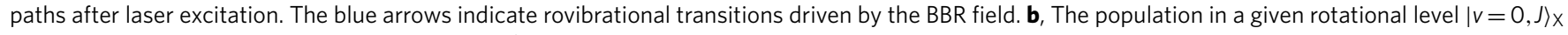
is determined by two-colour $1+1 \mathrm{REMPD}$ of $\mathrm{MgH}^{+}$. The state-selective excitation $|v=0, J\rangle_{X}-|v=0, J+1\rangle_{\mathrm{A}}(\mathrm{R}-\mathrm{branch})$ or $|v=0, J\rangle_{\mathrm{X}}-|v=0, J-1\rangle_{\mathrm{A}}$ (P-branch) around $281 \mathrm{~nm}$ is marked by the blue arrow, and the dissociating excitation to the $\mathrm{C}^{1} \Sigma^{+}$potential at $266 \mathrm{~nm}$ is marked by the purple arrow. $a_{0}$ represents the Bohr radius.

a

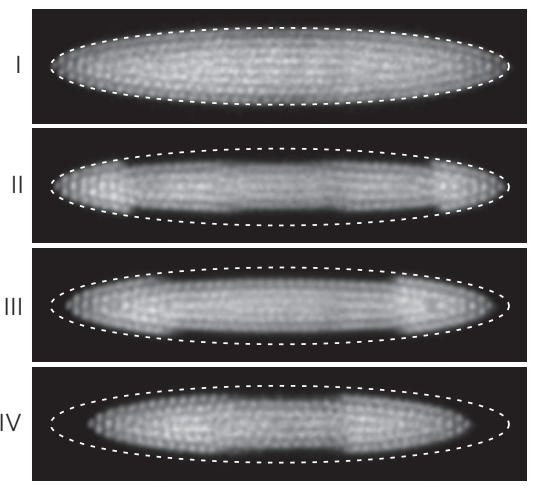

b

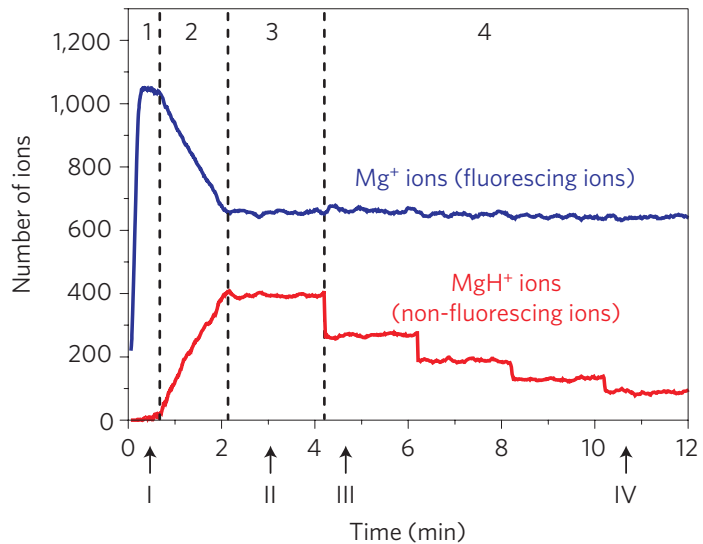

Figure 2 | Experimental sequence. a, Projection images of Coulomb crystals recorded by a CCD (charge-coupled device) camera: after Mg+ ion loading (I), during equilibration after formation of $\mathrm{MgH}^{+}$ions (II), and after one (III) and four (IV) REMPD events. The dashed ellipses indicate the contour of the initial crystal (I). b. The number of $\mathrm{Mg}^{+}$(blue curve) and $\mathrm{MgH}^{+}$(red curve) ions deduced from projection images of Coulomb crystals (for details see ref. 13). The intervals separated by the dashed vertical lines correspond to: trapping and cooling of $\mathrm{Mg}^{+}(1)$, formation of $\mathrm{MgH}^{+}(2)$, equilibration in the

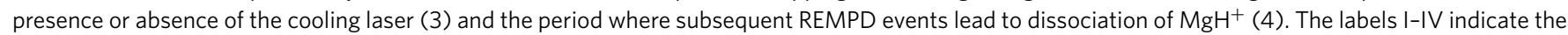
times at which the images in a were recorded.

at a partial pressure of $1 \times 10^{-9}$ mbar. When the desired number of molecular ions is produced (typically about 400 ions), a valve is closed and the partial pressure of $\mathrm{H}_{2}$ is reduced within $20 \mathrm{~s}$ to a level below $10^{-10}$ mbar using an ion getter pump (the total pressure is then $2-3 \times 10^{-10}$ mbar). As shown in Fig. 2 a (II), the formed $\mathrm{MgH}^{+}$ions are sympathetically cooled by the remaining $\mathrm{Mg}^{+}$ ions into a two-species Coulomb crystal, with the non-fluorescing $\mathrm{MgH}^{+}$ions positioned radially outside the fluorescing $\mathrm{Mg}^{+}$ions. The radial separation is caused by the slight mass difference between the two ion species ${ }^{23,27}$. The ion densities of $\mathrm{Mg}^{+}$and $\mathrm{MgH}^{+}$are, however, almost identical $\left(\rho_{\mathrm{Mg}^{+}}=10.2(4) \times 10^{7} \mathrm{~cm}^{-3}\right.$ and $\left.\rho_{\mathrm{MgH}^{+}}=9.8(4) \times 10^{7} \mathrm{~cm}^{-3}\right)$. As practically no loss of ions happens during the reaction period, the total ion number is furthermore unchanged. The numbers of $\mathrm{Mg}^{+}$and $\mathrm{MgH}^{+}$ions in a two-component Coulomb crystal can therefore rather easily be determined from projection images, such as the one presented in Fig. 2a (II), by assuming that the spheroidal outer shape of the two-component crystal is identical to the one in Fig. 2a (I).

After the valve has been closed, the $\mathrm{MgH}^{+}$ions are allowed to equilibrate internally for a period of two minutes in the presence of the room-temperature BBR field and optionally with a PbSe diode laser beam applied for cooling, by excitation of the $|v=0, J=2\rangle_{\mathrm{X}}-|v=1, J=1\rangle_{\mathrm{X}}$ transition at $1,609 \mathrm{~cm}^{-1}$. Both in the presence and absence of the rotational cooling beam, ample time is available to reach the rotational steady-state distribution. During the equilibration period the numbers of $\mathrm{Mg}^{+}$and $\mathrm{MgH}^{+}$ ions remain constant, as can be seen from region 3 in Fig. $2 b$.

After equilibration, two laser pulses at $266 \mathrm{~nm}$ and $281 \mathrm{~nm}$, respectively, are applied simultaneously to carry out a REMPD event. The pulses are generated by frequency quadrupling light 
a

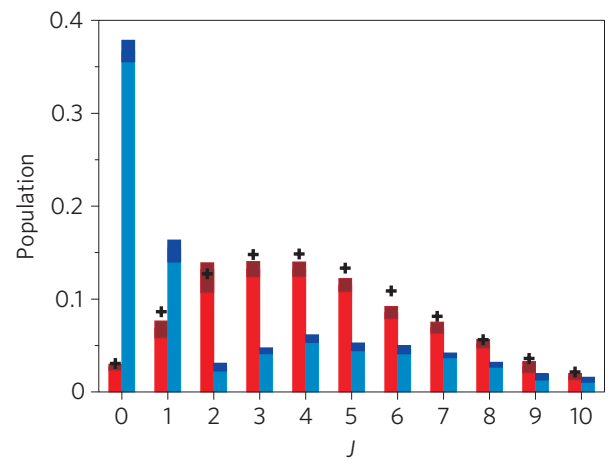

c

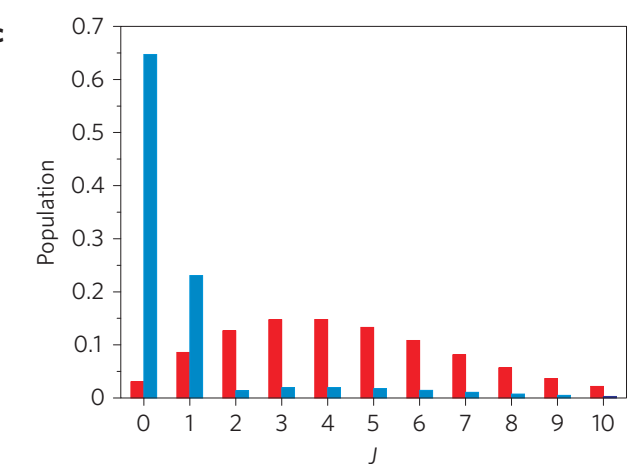

b

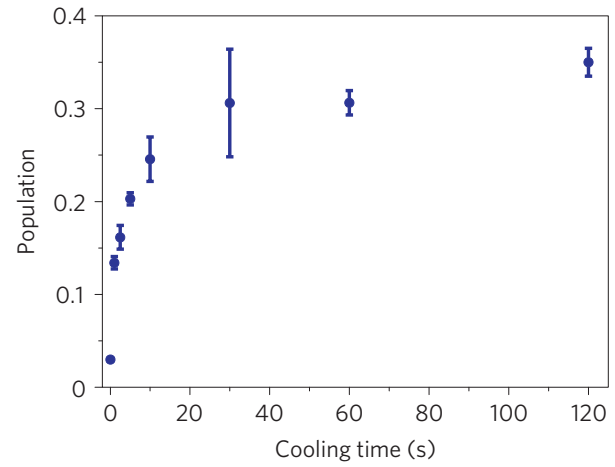

d

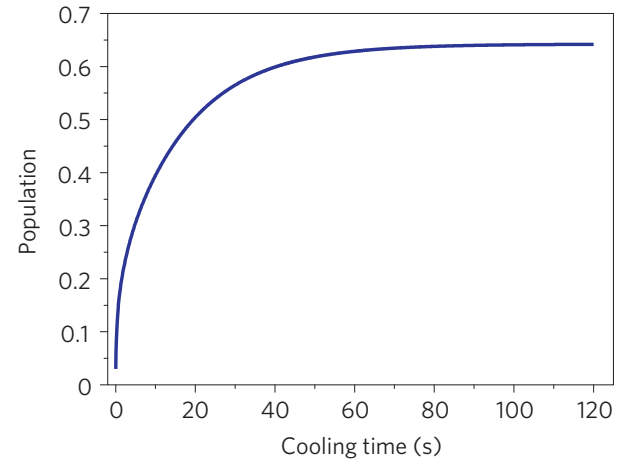

Figure 3 | Rotational cooling results. a, Deduced rotational state distributions obtained from the REMPD measurements. The red bars indicate the results without application of the laser cooling beam and the blue bars indicate the distribution after a two-minute cooling period. For comparison, a thermal

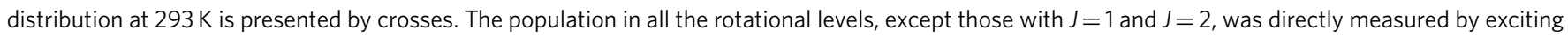
the $|v=0, J\rangle_{X}-|v=0, J+1\rangle_{A}$ transition (R-branch) in the REMPD event. As the populations in levels with $J=1$ and $J=2$ were not spectroscopically resolvable using R-branch transitions (see for example, ref. 13), P-branch transitions $|v=0, J\rangle_{X s c s \mid}-|v=0, J-1\rangle_{A}$ were used instead. To account for the fact that only $1 / 3$ and $3 / 5$ of the population in the levels with $J=1$ and $J=2$, respectively, is expected to be excited, owing to the fixed polarization of the

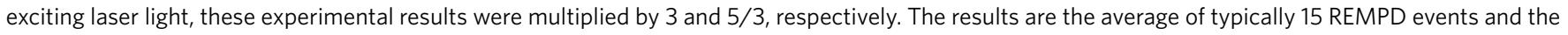
darker colours at the top of the bars indicates the \pm 1 standard deviation error. $\mathbf{b}$, The temporal evolution of the population in the ground state during rotational laser cooling. The population is clearly seen to saturate after one minute. The error bars are \pm 1 standard deviation. $\mathbf{c}$, Theoretical prediction of rotational state distributions. The red bars correspond to a thermal distribution at $293 \mathrm{~K}$ and the blue bars indicate the cooled distribution based on the model described in detail in ref. 13. d, The temporal evolution of the population in the ground state during rotational laser cooling, based on the model presented in ref. 13.

from a Nd:YAG laser $(266 \mathrm{~nm})$ and frequency doubling light from a dye laser pumped by the frequency doubled output from the same Nd:YAG laser $(281 \mathrm{~nm})$. The lengths of both pulses are $9 \mathrm{~ns}$ (FWHM).

When the pulse energies are set to $5 \mu \mathrm{J}$ and $1 \mathrm{~mJ}$ for the $281 \mathrm{~nm}$ and the $266 \mathrm{~nm}$ pulses, respectively, a single REMPD event is found to dissociate more than $90 \%$ of the $\mathrm{MgH}^{+}$ions in the rotational state addressed by the tunable $281 \mathrm{~nm}$ laser without significant off-resonant dissociation from other states (total of less than $2 \%$ ). In region 4 of Fig. $2 b$, an example of the stepwise decrease in the number of rotationally cooled $\mathrm{MgH}^{+}$ions in the ground state $|v=0, J=0\rangle_{\mathrm{X}}$ after successive REMPD events is shown. In Fig. 2a (III) and (IV) corresponding projection images are presented for reference. The combination of essentially perfect rotational level selectivity and nearly $100 \%$ dissociation probability in a single REMPD event provides a direct measure of the rotational state distribution at any chosen instant.

In Fig. 3a, the rotational state distributions for the 11 lowestlying levels are presented both for the situation without cooling and for the case where cooling has been applied for two minutes. Here, the directly measured populations in the 11 rotational levels, both before and after cooling is applied, sum up to about $90 \%$ within an uncertainty of only $\sim 5 \%$.

Whereas the uncooled distribution fits well with a thermal distribution at room temperature $(293 \mathrm{~K})$, the cooled distribution shows a pronounced accumulation of population in the two lowest rotational states. More precisely, $36.7 \pm 1.2 \%$ of the population is found in the $|v=0, J=0\rangle_{\mathrm{X}}$ state, $15.2 \pm 1.2 \%$ in the $|v=0, J=1\rangle_{\mathrm{X}}$ level and the remaining population is distributed among the $|v=0, J \geq 2\rangle_{\mathrm{X}}$ levels, with at most $6 \%$ in a single level. The fraction of molecules in the rovibrational ground state is equivalent to that of a thermal rotational distribution at $21.4 \pm 0.8 \mathrm{~K}$.

In Fig. $3 \mathrm{~b}$, the temporal evolution of the population in the rotational ground state during cooling is shown. Here, the ground-state population is observed to saturate after $40-60$ s. For comparison, theoretical predictions presented in ref. 13, based on a simplified rate equation model, are reproduced in Fig. $3 \mathrm{c}$,d. Besides spontaneous emission and the laser-driven cooling transition, this model includes all dipole-allowed transitions driven by BBR between the levels $|v=0, J=0 \ldots 20\rangle_{\mathrm{X}}$ and $|v=1, J=0 \ldots 20\rangle_{\mathrm{X}}$. Clearly, there is good agreement between the experimental and model results with respect to the timescale for the rotational cooling process as well as to the qualitative features of the cooled rotational state distribution. This fact supports that the essential physical processes are indeed included in the model. The quantitative difference between the experimentally obtained ground-state population and the model result may have several origins, including slight differences between the experimental conditions and the assumptions of the model, non-exact calculated values for the transition rates applied in the model and finally the rate model might be too simplistic. These potential reasons for the difference are being investigated for optimization of the present 
cooling scheme, as well as more complex rotational cooling schemes involving two or more cooling lasers (see also ref. 28).

The simplicity of the cooling scheme makes it generally applicable to a broad range of diatomic polar molecular ions with ${ }^{1} \Sigma$ electronic ground-state potentials. For this class of molecules, the only requirement for significant rotational cooling is that the $|v=1, J=1\rangle_{\mathrm{X}} \rightarrow|v=0, J=0\rangle_{\mathrm{X}}$ spontaneous transition rate has to greatly exceed the BBR-stimulated rate of the $|v=0, J=0\rangle_{\mathrm{X}} \rightarrow|v=0, J=1\rangle_{\mathrm{X}}$ transition. This will typically be the case for ionic hydrides, owing to their large vibrational constants.

Although the radiofrequency-field-driven coherent motional energy of the rotationally cooled molecular ions in the Coulomb crystals typically exceeds the thermal motion corresponding to $\sim 10 \mathrm{mK}$, (see refs 11, 27), for uni-molecular reaction studies involving coherent light fields, the effect of this motion can be avoided by propagating the light beams along the central radiofrequency-field-free axis of the trap. However, to carry out bimolecular reactions at low kinetic energies one will have to confine the molecular ions to the radiofrequency-field-free axis. In the case of many molecular ions, this can best be achieved by embedding a single string of molecular ions in a two-species crystal (for example, $\mathrm{MgH}^{+}$ions inside $\mathrm{aCa}^{+}$crystal $\left.^{29}\right)$. Alternatively, one can turn to the ultimate situation of having a single molecular ion sympathetically cooled by a single atomic ion and localized within a volume below $1 \mu \mathrm{m}^{3}$ centred at the radiofrequency-field-free axis ${ }^{30,31}$.

This last situation, combined with the demonstrated rotational cooling technique, gives unprecedented control in ionic uni- and bi-molecular reactions and will surely lead to improved molecular spectroscopy. Molecular ions initially prepared in their rovibrational ground state may even be exploited as physical quantum bits for an ion-trap-based quantum computer in the future.

\section{Received 28 August 2009; accepted 27 January 2010;} published online 7 March 2010

\section{References}

1. Koelemeij, J. C. J., Roth, B., Wicht, A., Ernsting, I. \& Schiller, S. Vibrational spectroscopy of $\mathrm{HD}^{+}$with 2-ppb accuracy. Phys. Rev. Lett. 98, 173002 (2007).

2. Hudson, J. J., Sauer, B. E., Tarbutt, M. R. \& Hinds, E. A. Measurement of the electron electric dipole moment using YbF molecules. Phys. Rev. Lett. 89, 023003 (2002).

3. Hudson, E. R., Lewandowski, H. J., Sawyer, B. C. \& Ye, J. Cold molecule spectroscopy for constraining the evolution of the fine structure constant. Phys. Rev. Lett. 96, 143004 (2006).

4. DeMille, D. Quantum computation with trapped polar molecules. Phys. Rev. Lett. 88, 067901 (2002).

5. André, A. et al. A coherent all-electrical interface between polar molecules and mesoscopic superconducting resonators. Nature Phys. 2, 636-642 (2006).

6. Santos, L., Shlyapnikov, G. V., Zoller, P. \& Lewenstein, M. Bose-Einstein condensation in trapped dipolar gases. Phys. Rev. Lett. 85, 1791-1794 (2000)

7. Büchler, H. P. et al. Strongly correlated 2D quantum phases with cold polar molecules: Controlling the shape of the interaction potential. Phys. Rev. Lett. 98, 060404 (2007).

8. Rice, S. A. \& Zaho, M. Optical Control of Molecular Dynamics (Wiley, 2000).

9. Shapiro, M. \& Brumer, P. W. Principles of the Quantum Control of Molecular Processes (Wiley, 2003)

10. Willitsch, S., Bell, M. T., Gingell, A. D. \& Softley, T. P. Chemical applications of laser- and sympathetically-cooled ions in ion traps. Phys. Chem. Chem. Phys. 10, 7200-7210 (2008).
11. Krems, R. V. Cold controlled chemistry. Phys. Chem. Chem. Phys. 10, 4079-4092 (2008).

12. Smith, I. W. M. Low Temperatures and Cold Molecules (Imperial College Press, 2008).

13. Højbjerre, K., Hansen, A. K., Skyt, P. S., Staanum, P. F. \& Drewsen, M. Rotational state resolved photodissociation spectroscopy of translationally and vibrationally cold $\mathrm{MgH}^{+}$ions: Toward rotational cooling of molecular ions. New J. Phys. 11, 055026 (2009).

14. Vogelius, I. S., Madsen, L. B. \& Drewsen, M. Blackbody-radiation-assisted laser cooling of molecular ions. Phys. Rev. Lett. 89, 173003 (2002).

15. Weinstein, J. D., deCarvalho, R., Guillet, T., Friedrich, B. \& Doyle, J. M. Magnetic trapping of calcium monohydride molecules at millikelvin temperatures. Nature 395, 148-150 (1998).

16. van de Meerakker, S. Y. T., Bethlem, H. L. \& Meijer, G. Taming molecular beams. Nature Phys. 4, 595-602 (2008).

17. Rieger, T., Junglen, T., Rangwala, S. A., Pinkse, P. W. \& Rempe, G. Continuous loading of an electrostatic trap for polar molecules. Phys. Rev. Lett. 95, 173002 (2005).

18. Hogan, S. D., Seiler, Ch. \& Merkt, F. Rydberg-state-enabled deceleration and trapping of cold molecules. Phys. Rev. Lett. 103, 123001 (2009).

19. Ni, K.-K. et al. A high phase-space-density gas of polar molecules. Science 322, 231-235 (2008).

20. Deiglmayr, J. et al. Formation of ultracold polar molecules in the rovibrational ground state. Phys. Rev. Lett. 101, 133004 (2008).

21. Viteau, M. et al. Optical pumping and vibrational cooling of molecules. Science 321, 232-234 (2008).

22. Gerlich, D. Ion-neutral collisions in a 22-pole trap at very low energies. Phys. Scr. T59, 256-263 (1995).

23. Mølhave, K. \& Drewsen, M. Formation of translationally cold $\mathrm{MgH}^{+}$and $\mathrm{MgD}^{+}$molecules in an ion trap. Phys. Rev. A 62, 011401(R) (2000).

24. Bertelsen, A., Jørgensen, S. \& Drewsen, M. The rotational temperature of polar molecular ions in Coulomb crystals. J. Phys. B 39, L83-L89 (2006).

25. Koelemeij, J. C. J., Roth, B. \& Schiller, S. Blackbody thermometry with cold molecular ions and application to ion-based frequency standards. Phys. Rev. A 76, 023413 (2007).

26. Vogelius, I. S., Madsen, L. B. \& Drewsen, M. Probabilistic state preparation of a single molecular ion by projection measurement. J. Phys. B 39, S1259-S1265 (2006).

27. Drewsen, M., Brodersen, C., Hornekær, L., Hangst, J. S. \& Schiffer, J. P. Large ion crystals in a Linear Paul Trap. Phys. Rev. Lett. 81, 2878-2881 (1998).

28. Schiller, S. et al. All-optical preparation of molecular ions in the rovibrational ground state. Nature Phys. doi:10.1038/nphys1605 (2010).

29. Hornekær, L., Kjærgaard, N., Thommesen, A. M. \& Drewsen, M. Structural properties of two-component coulomb crystals in Linear Paul Traps. Phys. Rev. Lett. 86, 1994-1997 (2001).

30. Drewsen, M., Mortensen, A., Martinussen, R., Staanum, P. \& Sørensen, J. L. Nondestructive identification of cold and extremely localized single molecular ions. Phys. Rev. Lett. 93, 243201 (2004).

31. Staanum, P. F., Højbjerre, K., Wester, R. \& Drewsen, M. Probing isotope effects in chemical reactions using single ions. Phys. Rev. Lett. 100, 243003 (2008).

\section{Acknowledgements}

We gratefully acknowledge M. Shapiro for the loan of the pulsed Nd:YAG laser and the Lundbeck Foundation for a grant for the pulsed dye laser. P.F.S. and M.D. acknowledge support from the Danish Natural Science Research Council.

\section{Author contributions}

All authors contributed substantially to the work presented in this paper.

\section{Additional information}

The authors declare no competing financial interests. Reprints and permissions information is available online at http://npg.nature.com/reprintsandpermissions. Correspondence and requests for materials should be addressed to M.D. 